\title{
Inhibition of Tumor Growth and Sensitization to Sunitinib by RNA Interference Targeting Programmed Death-ligand 1 in Mouse Renal Cell Carcinoma RenCa Model
}

\author{
TAKUTO HARA $^{1}$, HIDEAKI MIYAKE ${ }^{2}$, NOBUYUKI HINATA ${ }^{1}$ and MASATO FUJISAWA ${ }^{1}$ \\ ${ }^{1}$ Division of Urology, Kobe University Graduate School of Medicine, Kobe, Japan; \\ ${ }^{2}$ Department of Urology, Hamamatsu University School of Medicine, Hamamatsu, Japan
}

\begin{abstract}
Background/Aim: There are several unresolved issues regarding the combined treatment with an immune checkpoint inhibitor and anti-angiogenic agent for renal cell carcinoma (RCC) patients. The purpose of this study was to address the inhibitory effects of programmed death-ligand 1 (PD-L1) expression on growth and sensitivity to sunitinib in the mouse RCC RenCa model. Materials and Methods: We established RenCa/sh-PD-L1 by transfecting RenCa cells with a plasmid carrying a short hairpin RNA targeted against $P D$ L1. The growth pattern of RenCa/sh-PD-L1 with or without sunitinib was compared to that of RenCa cells transfected with control plasmid alone (RenCa/Co). Results: No significant difference in growth or sensitivity to sinitinib was noted between RenCa/sh-PD-L1 and RenCa/Co cells in vitro. The tumor volume in mice subcutaneously injected with RenCa/shPD-L1 was significantly smaller than that with RenCa/Co. Treatment of mice bearing each tumor with sunitinib resulted in a significant reduction of the RenCa/sh-PD-L1 tumor compared to the RenCa/Co tumor. Moreover, infiltration by $\mathrm{CD}^{+} \mathrm{T}$ cells of RenCa/sh-PD-L1 tumors was significantly higher than that of RenCa/Co tumors, irrespective of treatment with sunitinib. Conclusion: Suppressed expression of PD-L1 could increase tumor-infiltrating $C D 8^{+} T$ cells and result in growth inhibition as well as enhanced sensitivity to sunitinib in the RenCa model.
\end{abstract}

Renal cell carcinoma (RCC), the most common malignancy of the adult kidney, is characterized by unique features, including a markedly high incidence of metastasis; that is, approximately

Correspondence to: Hideaki Miyake, Department of Urology, Hamamatsu University School of Medicine, 1-20-1 Handayama, Higashi-Ku, Hamamatsu 431-3192, Japan. Tel: +81 534352306, Fax: +81 534352305, e-mail: hideakimiyake@ hotmail.com

Key Words: Renal cell carcinoma, PD-L1, sunitinib, RenCa, CD8+ $\mathrm{T}$ cells. one-third of RCC patients have metastatic disease at diagnosis, and $20-40 \%$ of patients having localized RCC who received surgical treatment with a curative intent consequently experience metastatic spread (1). In the last decade, the therapeutic strategy for patients with advanced RCC has been revolutionized by the introduction of novel agents that target vascular endothelial growth factor (VEGF) and the mammalian target of rapamycin (2). Moreover, it has been shown that antibody blockade of major molecules mediating immune checkpoint pathways, such as programmed death-1 (PD-1), PD-ligand 1 (PD-L1) and cytotoxic T-lymphocyte antigen 4, is efficient in patients with various types of malignant tumors, and these new immune therapies have also become potential approaches against advanced RCC (3). In fact, major clinical guidelines currently recommend the use of immune checkpoint inhibitors as standard agents for treatment-naïve as well as previously-treated advanced RCC patients (4).

Despite recent progress in systemic therapy for patients with advanced RCC, the prognostic outcomes of these patients remain unsatisfactory (2). In recent years, to overcome this limitation, the efficacies of several types of combined therapy with multiple agents have been explored (5-8). Of these, the combination of an immune checkpoint inhibitor with an antiangiogenic agent has been demonstrated to have a significant prognostic benefit compared with treatment with an antiangiogenic agent alone in pivotal randomized clinical trials (RCTs) (7, 8). For example, Rini et al. have conducted a phase 3 RCT that randomly assigned 861 patients with previously untreated advanced clear cell RCC into groups receiving either pembrolizumab plus axitinib or sunitinib alone, and demonstrated that treatment with pembrolizumab plus axitinib resulted in significantly longer overall and progression-free survivals that those with sunitinib (7). However, a number of issues with respect to combined treatment with an immune checkpoint inhibitor and an anti-angiogenic agent remain unresolved, including clarification of the optimal combination of agents and their dosing-schedules, as well as the mechanism involved in the anti-tumor activity (9). 
Considering these findings, we assessed the impact of PDL1 blocking in mouse RCC RenCa model on the growth and sensitivity to molecular-targeted agents both in vitro and in vivo in order to achieve new insights into the development of active anti-PD-L1-based therapy combined with a targeted agent.

\section{Materials and Methods}

Tumor cell line. RenCa cells, a mouse RCC cell line originating from of BALB/c, which were purchased from ATCC (Manassas, VA, USA), were cultured with MEM (Life Technologies Inc., Gaithersburg, MD, USA) containing $10 \%$ calf serum.

Expression plasmid and introduction to RenCa cells. A chemical synthetic oligodeoxynucleotide encoding a short hairpin RNA (shRNA) targeting mouse $P D-L 1$ was inserted downstream of the U6 promoter of the pGFP-V-RS Vector (ORIGENE, Rockville, MD, USA). Similarly, a control vector was generated by randomizing the sequence of mouse $P D-L 1$.

Plasmids were transfected into RenCa cells by liposome-mediated transfer method (10). Briefly, either the expression vector with shRNA targeted against mouse $P D-L 1$ or the control vector was administered to RenCa cells following incubation for $20 \mathrm{~min}$ with Lipofectamine $^{\mathrm{TM}}$ and OPTI-MEM (Invitrogen Life Technologies Inc.). Selection in $1 \mathrm{mg} / \mathrm{ml}$ puromycin (Sigma-Aldrich, St Louis, MO, USA) was started $72 \mathrm{~h}$ after transfection and then colonies were harvested and cell lines were established.

Proliferation assay. To evaluate the growth of RenCa sublines, $5 \times 10^{4}$ cells were seeded in 12-well plates. The number of cells was calculated by Cell Counting Kit-8 (Dojindo Molecular Technologies, Kumamoto, Japan). In addition, the sensitivity of RenCa sublines to sunitinib, temsirolimus (Sigma-Aldrich), sorafenib (Toronto Research Chemicals, Toronto, Canada) or everolimus (LC Laboratories, Woburn, MA, USA) was measured following $48 \mathrm{~h}$ of incubation with the targeted agent. Each experiment was conducted in triplicate.

Western blotting. Western blotting was carried out as previously reported (11). Samples containing equal amounts of protein $(25 \mu \mathrm{g})$ from the RenCa sublines were subjected to SDS-polyacrylamide gel electrophoresis and transferred to a nitrocellulose membrane (Schleicher \& Schuell BioScience Inc., Keene, NH, USA). The membranes were incubated overnight at $4^{\circ} \mathrm{C}$ with antibodies against mouse PD-L1, p53, VEGF receptor, phosphorylated (p)-VEGFR, cKit, Akt, p-Akt, Stat-3, p-Stat-3 (Cell Signaling Technology, Inc., Tokyo, Japan) and $\beta$-actin (Sigma-Aldrich). The membranes were then exposed for $30 \mathrm{~min}$ to secondary antibodies (Amersham Pharmacia Biotech, Arlington Heights, IL, USA), and specific proteins were visualized by a chemiluminescence system (Amersham Pharmacia Biotech).

Assessment of in vivo tumor growth. The BALB/c-+/+ male mice (5-6 weeks old) were purchased from Clea Japan (Tokyo, Japan), and housed in a controlled environment. Each animal experiment group consisted of 5 mice. The tumor cells of each RenCa subline were trypsinized, and $5 \times 10^{6}$ cells were injected subcutaneously with $100 \mu \mathrm{l}$ of Matrigel (Becton Dickinson, Franklin Lakes, NJ, USA). At 10 days after the implantation of tumor cells, mice were randomly assigned to treatment with sunitinib at a dose of $20 \mathrm{mg} / \mathrm{kg}$ or vehicle once daily for 2 weeks. Size of subcutaneous tumor was measured at least once weekly with calipers and calculated using the previously reported formula (12).

Histological evaluation. Tumors were harvested from mice 2 weeks after the initiation of treatment with sunitinib or vehicle. Immunohistochemical study of tumor tissues was conducted as previously described (12). Briefly, formaldehyde-fixed paraffin sections were deparaffinized using xylene and hydrated in ethanol. Following the inactivation of endogenous peroxidase by hydrogen peroxidase in methanol, tissue sections were boiled in $0.01 \mathrm{M}$ citrate buffer for $10 \mathrm{~min}$ and stained with antibodies against mouse PD-L1, CD4 and CD8 (Cell Signaling Technology, Inc.). Sections were then exposed to biotinylated secondary $\operatorname{IgG}$ (Vector Laboratories, Burlingame, CA, USA) for $30 \mathrm{~min}$. After incubation in avidin-biotinperoxidase complex for $30 \mathrm{~min}$, samples were incubated with diaminobenzidine tetrahydrochloride solution and counterstained with hematoxylin.

Statistical analysis. Statview 5.0 software (Abacus Concepts Inc., Berkley, CA, USA) was used to carry out statistical examinations, and $p$-values of $<0.05$ were recognized significant. An unpaired $t$-test was employed to compare differences between the two groups, and all outcomes are presented as the mean $\pm \mathrm{SD}$.

\section{Results}

Expression status of PD-L1 in RenCa sublines. An expression plasmid with shRNA targeted against $P D-L 1$ or the control plasmid was introduced into RenCa cells, and following drug selection, numerous clones were expanded. Western blotting was subsequently conducted to examine PD-L1 protein expression status in parental $\mathrm{RenCa}(\mathrm{RenCa} / \mathrm{P})$, control plasmid-transfected $\mathrm{RenCa}$ ( $\mathrm{RenCa} / \mathrm{Co}$ ), and 3 clones transfected with the plasmid carrying PD-L1 shRNA (RenCa/sh-PD-L1\#1 to RenCa/sh-PD-L1\#3). As presented in Figure 1, PD-L1 protein was definitely detected in $\mathrm{RenCa} / \mathrm{P}$ and $\mathrm{RenCa} / \mathrm{Co}$ cells, whereas PD-L1 expression in the $3 \mathrm{PD}-$ L1 shRNA-transfected clones was markedly suppressed in comparison with those in $\mathrm{RenCa} / \mathrm{P}$ and $\mathrm{RenCa} / \mathrm{Co}$.

In the in vitro studies described below, similar outcomes were achieved in the PD-L1 shRNA-transfected clones (RenCa/shPD-L1\#1 to RenCa/sh-PD-L1\#3) or the control clone (RenCa/P and $\mathrm{RenCa} / \mathrm{Co}$ ); thus, only the data corresponding to $\mathrm{RenCa} / \mathrm{sh}-$ PD-L1\#1 and $\mathrm{RenCa} / \mathrm{Co}$ are shown.

In vitro proliferation and expression of key molecules mediating tumor progression in RenCa sublines. To investigate the impact of inhibited PD-L1 expression on the proliferation of RenCa, proliferation of RenCa sublines was analyzed. There was no significant difference in the proliferation rates between $\mathrm{RenCa} / \mathrm{Co}$ and $\mathrm{RenCa} / \mathrm{sh}-\mathrm{PD}-\mathrm{L} 1 \# 1$ (Figure 2A).

Expression levels of several key molecules involved in tumor progression among RenCa sublines were subsequently compared. There was no significant difference in the 


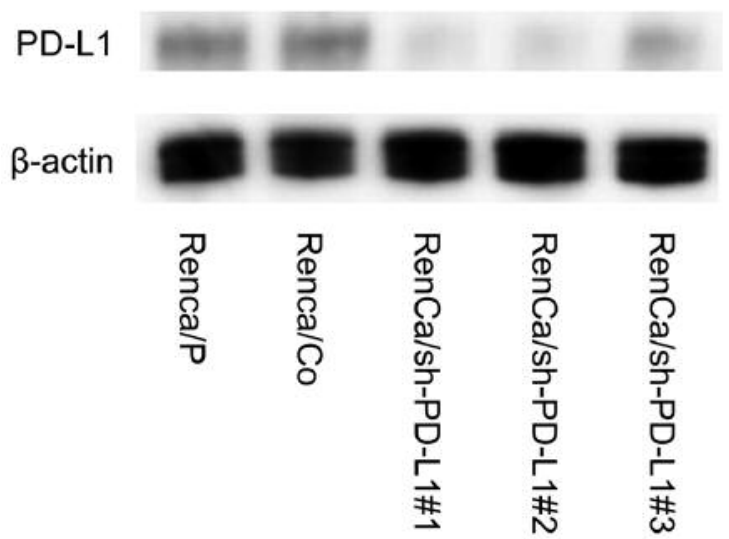

Figure 1. Expression levels of programmed death-ligand 1 (PD-L1) in RenCa sublines. (RenCa/P, parental cell line; RenCa/Co, control plasmid-only transfected cell line; RenCa/sh-PD-L1\#1 to \#3, PD-L1 short hairpin RNA-transfected cell lines). Protein was extracted and western blot analysis was conducted to examine the expression levels of PD-L1 and $\beta$-actin in each cell line.

expression levels of p53, VEGFR, p-VEGFR, c-Kit, Akt, pAkt, Stat-3 or p-Stat-3 between $\mathrm{RenCa} / \mathrm{Co}$ and $\mathrm{RenCa} / \mathrm{sh}-\mathrm{PD}$ L1\#1 (data not shown).

Sensitivity of RenCa subline to sunitinib. To assess the effect of PD-L1 on the sensitivity of RenCa to sunitinib, RenCa sublines were exposed to several doses of sunitinib. There was no significant difference in the sensitivity to sunitinib between $\mathrm{RenCa} / \mathrm{Co}$ and RenCa/sh-PD-L1\#1 (Figure 2B). Moreover, no significant difference in the sensitivity to sorafenib, everolimus or temsirolimus was observed between these two cell lines (data not shown).

In vivo growth of RenCa sublines with and without sunitinib treatment. To assess in vivo growth rates of RenCa sublines with and without administration of sunitinib, $5 \times 10^{6}$ cells of each clone were injected into syngeneic mice. As shown in Figure 3, growth of RenCa/sh-PD-L1\#1 tumors in mice without sunitinib administration was significantly slower than that of $\mathrm{RenCa} / \mathrm{Co}$ tumors. Furthermore, despite the definitive growth inhibition of both RenCa sublines by treatment with sunitinib, a significantly higher growth suppressive effect of sunitinib on RenCa/sh-PD-L1\#1 tumors was observed compared with that on $\mathrm{RenCa} / \mathrm{Co}$ tumors; that is, the size of $\mathrm{RenCa} / \mathrm{Co}$ tumors increased twofold compared with that of RenCa/sh-PD-L1\#1 tumors after the completion of sunitinib treatment (Figure 3).

Assessment of microenvironment of RenCa sublines with and without sunitinib therapy. To investigate the microenvironment around in vivo tumors derived from RenCa sublines, tumor
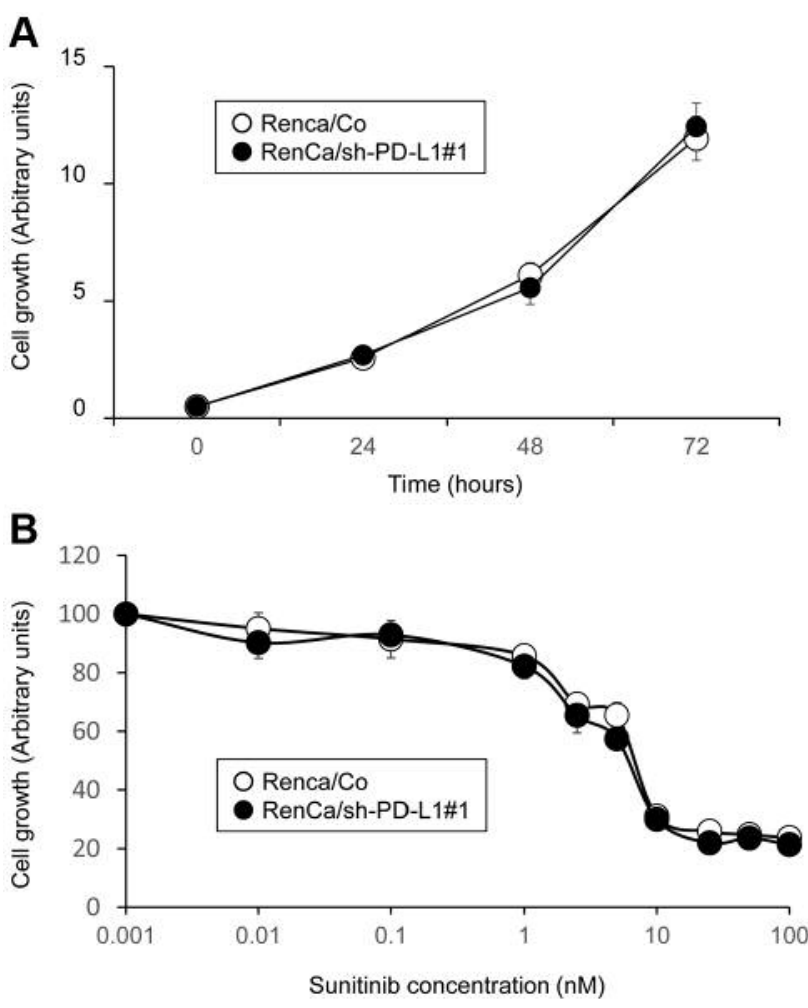

Figure 2. Growth patterns of RenCa sublines with or without sunitinib. (A) Growth rates of RenCa sublines. In vitro growth of RenCa/Co and RenCa/sh-PD-L1\#1 were evaluated by measuring the cell number of each cell line in triplicate. Bars, standard deviations. (B) Impact of administration of sunitinib on growth of RenCa sublines. RenCa/Co and RenCa/sh-PD-L1\#1 were exposed to the indicated doses of sunitinib. Following 48 h of treatment, growth rate was assessed in triplicate by measuring the cell number of each cell line in 3 independent experiments. Bars, standard deviations.

tissues were immunohistochemical examined (Figure 4). We initially confirmed that PD-L1 expression was not detected in RenCa/sh-PD-L1\#1 tumors, while PD-L1 was definitely expressed in RenCa/Co tumors. Furthermore, irrespective of treatment with sunitinib, significantly higher infiltration of $\mathrm{CD}^{+} \mathrm{T}$ cells was detected in RenCa/sh-PD-L1\#1 tumors compared with $\mathrm{RenCa} / \mathrm{Co}$ tumors; however, no significant difference in the infiltration of $\mathrm{CD} 4^{+} \mathrm{T}$ cells was noted between these two tumors.

\section{Discussion}

The therapeutic strategy for advanced RCC has markedly changed over the last decade due to the wide spread of molecular-targeted agents and immune checkpoint inhibitors; however, it remains difficult to completely eradicate advanced RCC with systemic therapy using such types of agents (2). Accordingly, it has been intensively investigated whether 


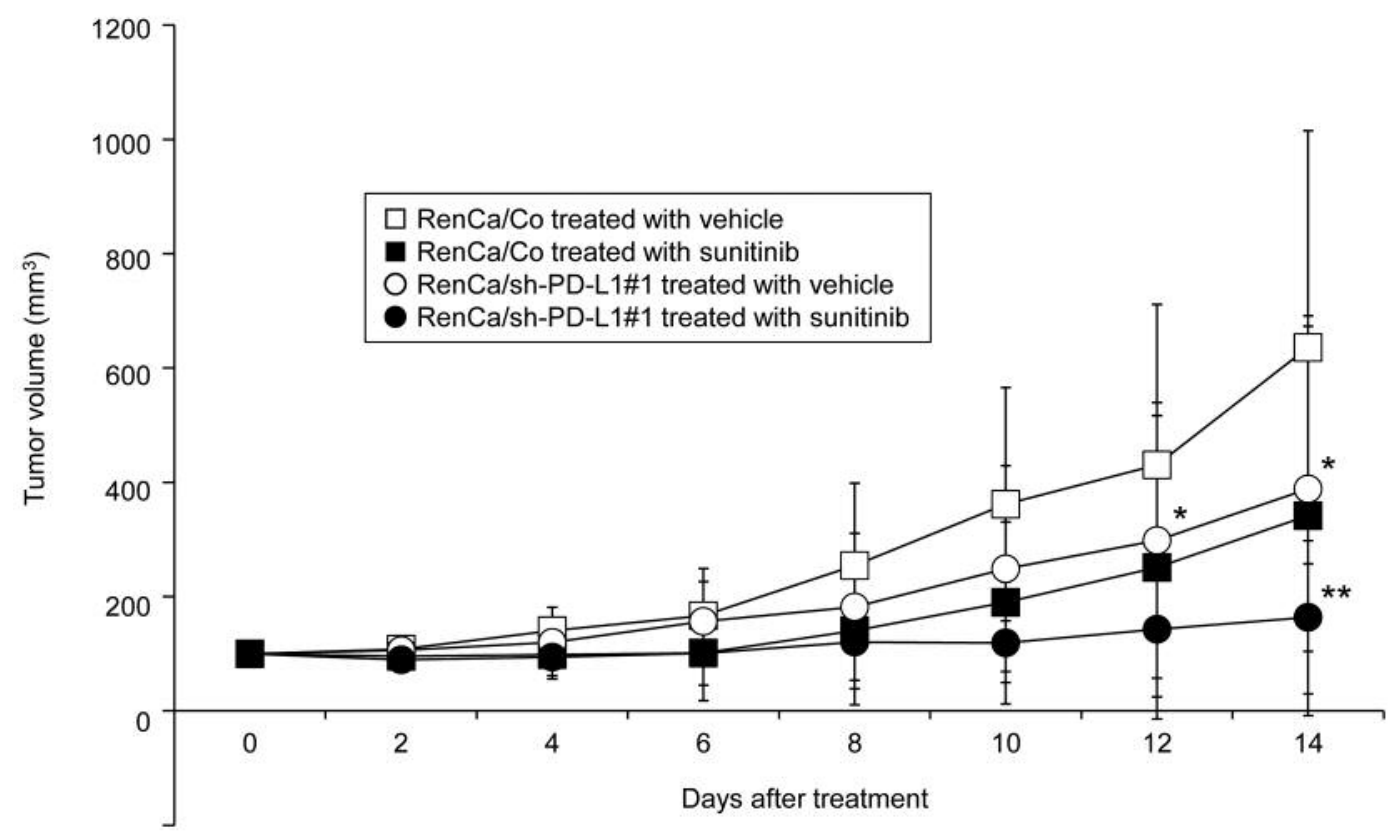

Figure 3. Impact of sunitinib treatment on the growth of RenCa sublines. Subcutaneous injection of $5 \times 10^{6}$ cells of each RenCa subline was carried out in 20 mice, which were randomized into those that received $20 \mathrm{mg} / \mathrm{kg}$ sunitinib and those that received, vehicle 5 times per week for 4 weeks. Tumor volume was measured over time. Bars, standard deviations. * and **, difference from RenCa/Co treated with vehicle and with sunitinib, respectively $(p<0.05)$.

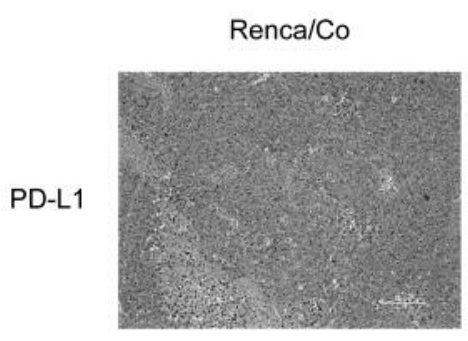

Renca/sh-PD-L1\#1

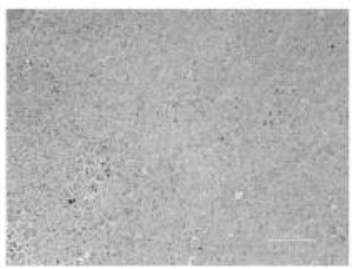

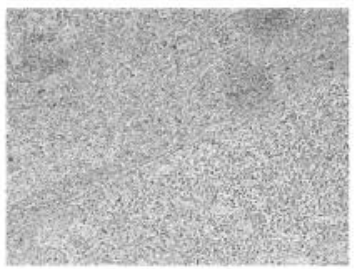

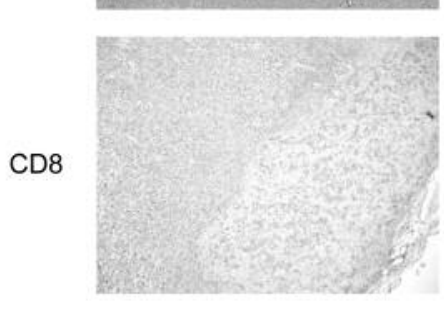

Sunitinib

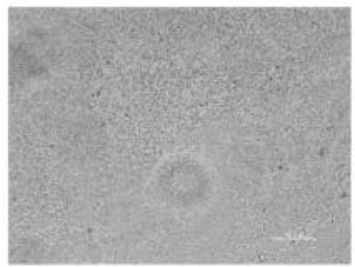

CD4

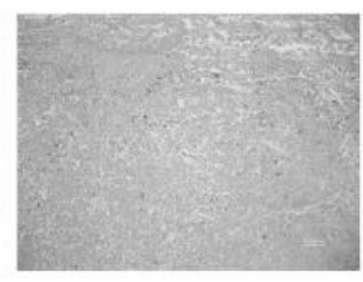

$(-)$
Renca/Co
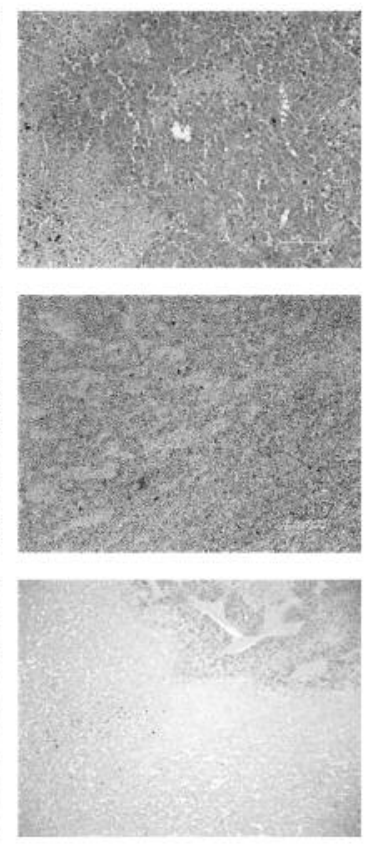

$(+)$
Renca/sh-PD-L1\#1
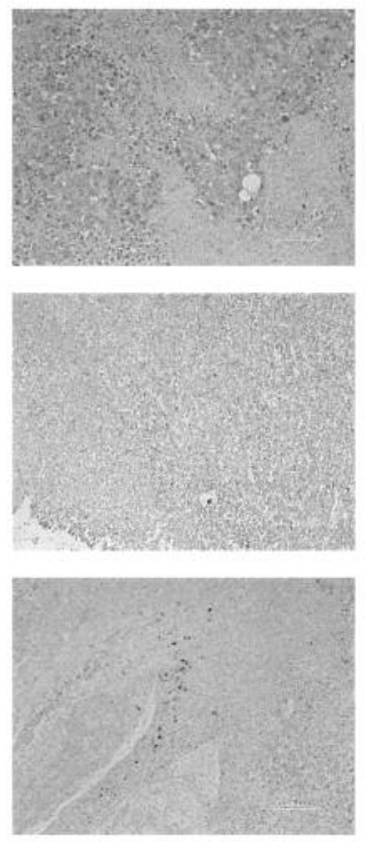

Figure 4. Histological examination of RenCa tumors after sunitinib treatment. Subcutaneous tumors were harvested from mice after administration of sunitinib or vehicle for 5 weeks based on the schedule shown above. Each tumor specimen was analyzed by immunohistochemical staining with antibodies against programmed death-ligand 1 (PD-L1), CD4 and CD8. 
combined treatments with these agents could further improve the prognosis of patients with advanced RCC (5). Of these therapies, the combination of an immune checkpoint inhibitor with an anti-angiogenic agent has been shown to provide a significant prognostic benefit within an acceptable safety profile compared with monotherapy using the anti-angiogenic agent alone $(7,8)$. Despite the promising clinical outcomes, several important issues associated with combined treatment with an immune checkpoint inhibitor and an anti-angiogenic agent remain unclarified, particularly optimal selection from multiple candidate agents (9). In this study, therefore, we investigated the inhibitory effect of the combination of antiPD-L1 therapy with sunitinib, one of the most effective antiangiogenic agents (13), on the growth of RCC cells both in vitro and in vivo using a mouse RCC RenCa model.

We initially established RenCa/sh-PD-L1 cells, in which PD-L1 expression was markedly inhibited using shRNA technology. On in vitro assessment, the growth of $\mathrm{RenCa} / \mathrm{sh}-$ PD-L1 cells was similar to that of Renca/Co cells. Furthermore, there were no significant differences in the expression profiles of major molecules mediating the progression of RCC between these two cell lines. PD-L1 has been demonstrated to play a potential role in immune evasion and $\mathrm{T}$ cell exhaustion (14). It has also been shown to be highly expressed in various types of malignant tumors, including RCC, and to be correlated with parameters suggesting an aggressive tumor phenotype (15-17). However, there have not been any studies clearly characterizing the mechanism involved in tumor progression directly regulated by PD-L1 beyond the immune system. Considering the findings of this study, one of the pressing research topics is to identify the molecular mechanism, other than immune modulation, by which PD-L1 promotes the progression of RCC in order to develop a new therapeutic approach for patients with advanced RCC. Therefore, we were interested in evaluating the inhibitory effect of PD-L1 on the sensitivity of the RenCa model to sunitinib. There were no significant differences in the in vitro sensitivity to sunitinib, in addition to that to other targeted agents, including sorafenib, everolimus and temsirolimus, between $\mathrm{RenCa} /$ sh-PD-L1 and RenCa/Co cells. Similarly, Hirayama et al. have previously reported that anti-mouse PD-L1 alone did not affect the in vitro growth of RenCa cells, and that antimouse PD-L1 did not enhance the in vitro sensitivity of RenCa to everolimus (18). Collectively, these findings suggest the absence of a significant impact of PD-L1 inhibition on the in vitro sensitivity to several targeted agents. We hypothesized that in vivo modulation of the immune environment might be required to realize the combined activity of an immune checkpoint inhibitor and a targeted agent against RCC. Therefore, we assessed the in vivo changes in the growth rates and sensitivity to sunitinib as well as their mechanisms in the RenCa model. The in vivo experiments of this study showed that the RenCa/sh-PD-L1 tumors were significantly smaller than $\mathrm{RenCa}$ /Co tumors, and that administration of sunitinib induced the significant reduction of the size of $\mathrm{RenCa} / \mathrm{sh}-\mathrm{PD}$ $\mathrm{L} 1$ tumors in comparison with that of $\mathrm{RenCa} / \mathrm{Co}$ tumors. These results suggest that inactivation of $\mathrm{PD}-\mathrm{L} 1$ is a promising approach in treating advanced RCC through the inhibition of tumor growth itself as well as sensitization to sunitinib. Furthermore, we also revealed that despite the lack of a difference in the infiltrating status of $\mathrm{CD}^{+} \mathrm{T}$ cells, the infiltration of $\mathrm{CD}^{+} \mathrm{T}$ cells in RenCa/sh-PD-L1 tumors was significantly higher than that in $\mathrm{RenCa} / \mathrm{Co}$ tumors, irrespective of sunitinib treatment. These outcomes indicate that inhibition of PD-L1 expression could induce cytotoxic $\mathrm{CD}^{+} \mathrm{T}$ cells, resulting in a marked reduction of the $\mathrm{RCC}$ tumor volume, particularly when combining treatment with sunitinib.

This study has several limitations. Initially, although PD-L1 expression was effectively inhibited in RenCa/sh-PD-L1, PD-L1 expression in host mice was not affected in the model used in this study. Therefore, the present in vivo findings may not fully reflect the effect of anti-PD-L1 therapy. Secondly, this study focused on the efficacy of sunitinib as a combined agent with PD-L1 inhibition; however, some other potential targeted agents against RCC are currently available. Finally, to date, there have been several studies showing the function of sunitinib as an immunomodulatory agent $(19,20)$. For example, Ko et al. have reported the ability of sunitinib to prevent myeloid-derived suppressor cell accumulation and restore normal T-cell functioning in mice bearing RenCa tumors (19). Thus, it may be possible to generate more useful information for the development of a combined therapeutic strategy with multiple agents against advanced RCC by further precise investigation of the immuno-modulatory role of anti-angiogenic agents.

\section{Conclusion}

These findings suggest that inhibition of PD-L1 expression can induce tumor-infiltrating $\mathrm{CD}^{+} \mathrm{T}$ cells and result in both growth suppression and enhanced efficacy of sunitinib in the mouse RCC RenCa model; accordingly, it may be of interest to further assess the impact of anti-PD-L1 treatment either alone or in combination with sunitinib as one of the novel alternative combination therapies for advanced RCC.

\section{Conflicts of Interest}

The Authors have no conflict of interest to declare regarding this study.

\section{Authors' Contributions}

The types of contribution by each author are as follows: Study conception and design, Hideaki Miyake, Masato Fujisawa; Acquisition of data, Takuto Hara; Analysis and interpretation of data, Nobuyuki Hinata, Hideaki Miyake; Drafting of manuscript, Takuto Hara, Hideaki Miyake. 


\section{Acknowledgements}

This study was partially supported by Pfizer Inc.

\section{References}

1 Rini BI, Rathmell WK and Godley P: Renal cell carcinoma. Curr Opin Oncol 20: 300-316, 2008. PMID: 18391630. DOI: 10.1097/CCO.0b013e3282f9782b

2 Bedke J, Gauler T, Grünwald V, Hegele A, Herrmann E, Hinz S, Janssen J, Schmitz S, Schostak M, Tesch H, Zastrow S and Miller $\mathrm{K}$ : Systemic therapy in metastatic renal cell carcinoma. World J Urol 35: 179-188, 2017. PMID: 27277600. DOI: 10.1007/s00345016-1868-5

3 Atkins MB, Clark JI and Quinn DI: Immune checkpoint inhibitors in advanced renal cell carcinoma: experience to date and future directions. Ann Oncol 28: 1484-1494, 2017. PMID: 28383639. DOI: $10.1093 /$ annonc/mdx 151

4 Ljungberg B, Albiges L, Abu-Ghanem Y, Bensalah K, Dabestani S, Fernández-Pello S, Giles RH, Hofmann F, Hora M, Kuczyk MA, Kuusk T, Lam TB, Marconi L, Merseburger AS, Powles T, Staehler M, Tahbaz R, Volpe A and Bex A: European Association of Urology Guidelines on renal cell carcinoma: the 2019 update. Eur Urol 75: 799-810, 2019. PMID: 30803729. DOI: 10.1016/ j.eururo.2019.02.011

5 George S, Rini BI and Hammers HJ: Emerging Role of combination immunotherapy in the first-line treatment of advanced renal cell carcinoma: a review. JAMA Oncol 5: 411-421, 2019. PMID: 30476955. DOI: 10.1001/jamaoncol.2018.4604

6 Motzer RJ, Tannir NM, McDermott DF, Arén Frontera O, Melichar B, Choueiri TK, Plimack ER, Barthélémy P, Porta C, George S, Powles T, Donskov F, Neiman V, Kollmannsberger CK, Salman P, Gurney H, Hawkins R, Ravaud A, Grimm MO, Bracarda S, Barrios CH, Tomita Y, Castellano D, Rini BI, Chen AC, Mekan S, McHenry MB, Wind-Rotolo M, Doan J, Sharma P, Hammers HJ and Escudier B: Nivolumab plus ipilimumab versus sunitinib in advanced renal-cell carcinoma. N Engl J Med 378: 1277-1290, 2018. PMID: 29562145. DOI: 10.1056/NEJMoa 1712126

7 Rini BI, Plimack ER, Stus V, Gafanov R, Hawkins R, Nosov D, Pouliot F, Alekseev B, Soulières D, Melichar B, Vynnychenko I, Kryzhanivska A, Bondarenko I, Azevedo SJ, Borchiellini D, Szczylik C, Markus M, McDermott RS, Bedke J, Tartas S, Chang YH, Tamada S, Shou Q, Perini RF, Chen M, Atkins MB and Powles T: Pembrolizumab plus axitinib versus sunitinib for advanced renal-cell carcinoma. N Engl J Med 380: 1116-1127, 2019. PMID: 30779529. DOI: 10.1056/NEJMoa1816714

8 Motzer RJ, Penkov K, Haanen J, Rini B, Albiges L, Campbell MT, Venugopal B, Kollmannsberger C, Negrier S, Uemura M, Lee JL, Vasiliev A, Miller WH Jr, Gurney H, Schmidinger M, Larkin J, Atkins MB, Bedke J, Alekseev B, Wang J, Mariani M, Robbins PB, Chudnovsky A, Fowst C, Hariharan S, Huang B, di Pietro A and Choueiri TK: Avelumab plus axitinib versus sunitinib for advanced renal-cell carcinoma. N Engl J Med 380: 1103-1115, 2019. PMID: 30779531. DOI: 10.1056/NEJMoa1816047

9 Escudier B: Combination therapy as first-Line treatment in metastatic renal-cell carcinoma. N Engl J Med 380: 1176-1178, 2019. PMID: 30779526. DOI: 10.1056/NEJMe1900887

10 Terakawa T, Miyake H, Furukawa J, Ettinger SL, Gleave ME and Fujisawa M: Enhanced sensitivity to androgen withdrawal due to overexpression of interleukin-6 in androgen-dependent human prostate cancer LNCaP cells. Br J Cancer 101: 1731-1739, 2009. PMID: 19844233. DOI: 10.1038/sj.bjc.6605358

11 Harada K, Miyake H, Kumano M and Fujisawa M: Acquired resistance to temsirolimus in human renal cell carcinoma cells is mediated by the constitutive activation of signal transduction pathways through mTORC2. Br J Cancer 109: 2389-2395, 2013. PMID: 24091619. DOI: 10.1038/bjc.2013.602

12 Kusuda Y, Miyake H, Gleave ME and Fujisawa M: Clusterin inhibition using OGX-011 synergistically enhances antitumour activity of sorafenib in a human renal cell carcinoma model. Br J Cancer 106: 1945-1952, 2012. PMID: 22588555. DOI: 10.1038/ bjc.2012.209

13 Wood L: Sunitinib malate for the treatment of renal cell carcinoma. Expert Opin Pharmacother 13: 1323-1336, 2012. PMID: 22607009. DOI: 10.1517/14656566.2012.689130

$14 \mathrm{Kyi} \mathrm{C}$ and Postow MA: Checkpoint blocking antibodies in cancer immunotherapy. FEBS Lett 588: 368-376, 2014. PMID: 24161671. DOI: $10.1016 /$ j.febslet.2013.10.015

15 Hara T, Miyake H and Fujisawa M: Expression pattern of immune checkpoint-associated molecules in radical nephrectomy specimens as a prognosticator in patients with metastatic renal cell carcinoma treated with tyrosine kinase inhibitors. Urol Oncol 35: 363-369, 2017. PMID: 28169111. DOI: 10.1016/j.urolonc. 2017.01.002

16 Joseph RW, Millis SZ, Carballido EM, Bryant D, Gatalica Z, Reddy S, Bryce AH, Vogelzang NJ, Stanton ML, Castle EP and Ho TH: PD-1 and PD-L1 expression in renal cell carcinoma with sarcomatoid differentiation. Cancer Immunol Res 3: 1303-1307, 2015. PMID: 26307625. DOI: 10.1158/2326-6066.CIR-15-0150

17 Rojkó L, Reiniger L, Téglási V, Fábián K, Pipek O, Vágvölgyi A, Agócs L, Fillinger J, Kajdácsi Z, Tímár J, Döme B, Szállási Z and Moldvay J: Chemotherapy treatment is associated with altered PD-L1 expression in lung cancer patients. J Cancer Res Clin Oncol 144: 1219-1226, 2018. PMID: 29675791. DOI: 10.1007/ s00432-018-2642-4

18 Hirayama Y, Gi M, Yamano S, Tachibana H, Okuno T, Tamada S, Nakatani $\mathrm{T}$ and Wanibuchi H: Anti-PD-L1 treatment enhances antitumor effect of everolimus in a mouse model of renal cell carcinoma. Cancer Sci 107: 1736-1744, 2016. PMID: 27712020. DOI: 10.1111/cas.13099

19 Ko JS, Rayman P, Ireland J, Swaidani S, Li G, Bunting KD, Rini B, Finke JH and Cohen PA: Direct and differential suppression of myeloid-derived suppressor cell subsets by sunitinib is compartmentally constrained. Cancer Res 70: 3526-3536, 2010. PMID: 20406969. DOI: 10.1158/0008-5472.CAN-09-3278

20 Najjar YG, Rayman P, Jia X, Pavicic PG Jr, Rini BI, Tannenbaum C, Ko J, Haywood S, Cohen P, Hamilton T, Diaz-Montero CM and Finke J: Myeloid-derived suppressor cell subset accumulation in renal cell carcinoma parenchyma is associated with intratumoral expression of IL1 $\beta$, IL8, CXCL5, and Mip- $1 \alpha$. Clin Cancer Res 23: 2346-2355, 2017. PMID: 27799249. DOI: 10.1158/10780432.CCR-15-1823 